\title{
Foetal and Neonatal Outcome in Pregnant Women with COVID-19
}

\section{Rupesh Shrestha, Srijana Basnet and Laxman Shrestha}

Department of Paediatrics, Tribhuvan University Teaching Hospital, Institute of Medicine, Kathmandu, Nepal

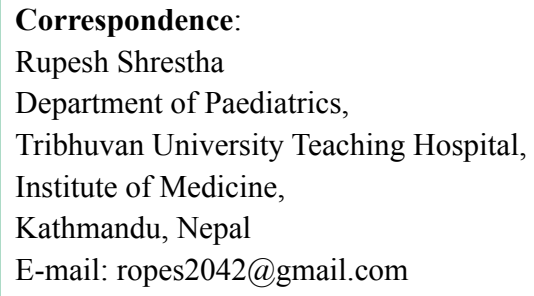

DOI: $10.3126 /$ jnps.v40i3.29844

Submitted on: $2020-07-03$

Accepted on: 2020-10-04

Acknowledgements: None

Funding: Nil

Conflict of Interest: None declared

Permission from IRB: Yes

To cite this article: Shrestha $\mathrm{R}$, Basnet S, Shrestha L. Fetal and Neonatal Outcome in Pregnant Women with COVID-19. J Nepal Paediatr Soc. 2020;40(3):148-56.

\section{ABSTRACT}

Introduction: The pandemic caused by coronavirus disease 2019 has adversely affected the health of all individuals including pregnant women. The susceptibility of pregnant women and their foetuses to severe acute respiratory syndrome coronavirus 2 infection is not clearly known. The objective of this review is to summarise the available evidence on foetal and neonatal outcomes of pregnant women with confirmed coronavirus disease 2019.

Methods: The review was conduced by searching the PubMed and MedRxiv databases for studies reporting outcome of pregnancy with confirmed coronavirus disease from December 2019 to April 2020 using combination of terms "pregnancy", "coronavirus disease 2019", "foetal outcome" and "neonatal outcome". Only peer reviewed articles reporting outcome of pregnancy with confirmed coronavirus disease 2019 were included in the review irrespective of language. Ongoing pregnancies, induced abortion, and pregnancies without outcome were excluded from analysis. Variables extracted and analysed included gestational age at delivery, mode of delivery, foetal and neonatal outcome.

Results: In total, 21 studies consisting 230 pregnant women including three twin pregnancies were enrolled in the study. Fever and cough were the most common symptoms reported in pregnant women. One hundred and sixty-two (70.4\%) pregnant women underwent cesarean section and there were $68(29.6 \%)$ preterm deliveries. Eight babies tested positive out of 161 newborns that were tested for coronavirus infection. Among adverse outcomes reported, there were two miscarriages, two still births and only one neonatal death.

Conclusions: Outcome of pregnancy with coronavirus disease 2019 in late trimester appears to be favourable. Occurrence of preterm delivery and cesarean section appear higher among infected pregnant women in comparison to general population. There was no conclusive evidence of vertical transmission.

Key Words: Coronavirus; Newborn; Pregnancy Outcome

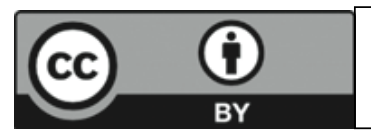

This work is licensed under creative common attribution 3.0 license 


\section{INTRODUCTION}

The growing pandemic of coronavirus disease 2019 (COVID-19) has posed a new threat to all humans worldwide with naive immune system to fight against it. COVID-19, which was first reported in Wuhan, China in December 2019, is caused by severe acute respiratory syndrome coronavirus 2 (SARS-CoV-2) belonging to the coronavirus genus. Coronaviruses (CoV) are enveloped, nonsegmented, positive sense single stranded ribonucleic acid (RNA) virus surrounded by a protein envelope, belonging to the family Coronaviridae. ${ }^{1}$ Clinical spectrum of COVID-19 ranges from asymptomatic and cold like symptoms to severe pneumonia, even causing multi-organ failure and death especially in elderly population and those with co-morbidities.

Pregnant women consist of typically vulnerable group to infectious diseases, not only because they have exaggerated disease manifestations but also due to detrimental impact on their obstetric and neonatal outcome. ${ }^{2}$ Altered cell-mediated immune functions make pregnant women more susceptible to infections. The physiological and anatomical changes leading to cardiopulmonary alternations in pregnant women further attenuate their ability to compensate for respiratory disorders. ${ }^{3}$ Moreover, infection in mother put their unborn foetus at high risk, particularly due to the immature innate and adaptive immune system. SARS (Severe Acute Respiratory Syndrome) and MERS (Middle East Respiratory Syndrome) caused by other viruses from the coronavirus family were associated with poorer outcomes during pregnancy like abortion, intrauterine foetal growth restriction, preterm delivery and maternal mortality. ${ }^{4}$ However, information about the outcome of pregnancy with COVID-19 is limited. Result is based on handful of case reports and series, with diverse findings. Definite evidence of vertical transmission of this infection has not been confirmed yet. There is also concern regarding role of neonates in transmission of infection to others. Understanding the implication of neonatal COVID-19 infection is critical in the development of guideline for this population.
The main objective of this review is to analyse the published literature reporting foetal and neonatal outcome of pregnant women with confirmed COVID-19 and provide the scientific information in a concise manner to guide the management and preventive care of COVID-19 in newborns.

\section{METHODS}

We conducted literature search using PubMed and MedRxiv from December 2019 to April 2020 using the following search items: "pregnancy", "coronavirus disease 2019", "foetal outcome" and "neonatal outcome". Among identified studies, peer reviewed full-text articles including original data were retrieved for inclusion criteria. Additional relevant studies were searched manually. Eligibility criteria included laboratory confirmed COVID-19 pregnant women with availability of information on pregnancy outcome: foetal and / or neonatal. Laboratory-confirmed case of COVID 19 was defined as a positive result on reverse-transcriptasepolymerase-chain-reaction (RT-PCR) on maternal respiratory tract specimen. No restriction regarding the publication language or date was applied. Exclusion criteria were: pregnancies with suspected COVID-19 that were not confirmed by the laboratory test, ongoing pregnancy, unreported foetal and neonatal outcomes, induced abortions. Review articles, recommendations, narratives, and study suspicious of duplicate reporting were excluded for analysis.

Articles were independently screened by authors for validity of inclusion criteria. All the shortlisted articles were read thoroughly by all three authors and inclusion of the article for the review was based on the consensus among the authors. Preferred Reporting Items for Systematic reviews and Meta-Analyses (PRISMA) guidelines were considered but not strictly adhered to.

Data on gestational age at delivery, mode of delivery, neonatal birth weight, Apgar scores, vertical transmission, newborn clinical presentations, foetal and neonatal outcome, neonatal intensive care unit (NICU) admissions and diagnostic tests was collected. To evaluate the degree of vertical transmission of COVID-19, subgroup analysis was carried out by compiling the 
data describing SARS-CoV-2 testing by RT-PCR in different maternal and neonatal samples.

Statistical analysis was done with SPSS version, 20. Variables were expressed as number of cases and percentages $(\%)$.

\section{RESULTS}

Twenty-one articles published from December 2019 through April 2020 reporting outcome on 230 pregnant women with COVID-19 including three twin pregnancies were selected for full-text review after screening and assessment of eligibility. The articles were composed of case reports $(\mathrm{n}=7)$, case series $(n=8)$, cohort studies $(n=3)$ and casecontrol studies $(\mathrm{n}=3)$. The majority of the studies were conducted in China $(\mathrm{n}=13),{ }^{5-17}$ but reports from United States $(\mathrm{n}=3),{ }^{2,18,19}$ Spain $(\mathrm{n}=1),{ }^{20}$ Italy $(\mathrm{n}=1),{ }^{21}$ Portugal $(\mathrm{n}=1),{ }^{22}$ Switzerland $(\mathrm{n}=$ $1),{ }^{23}$ and Canada $(\mathrm{n}=1)^{24}$ were also included. About half $(50.9 \%)$ of the total pregnant women with COVID-19 were from China.

The flow diagram of study selection process and foetal and neonatal outcome is summarised in Figure 1.

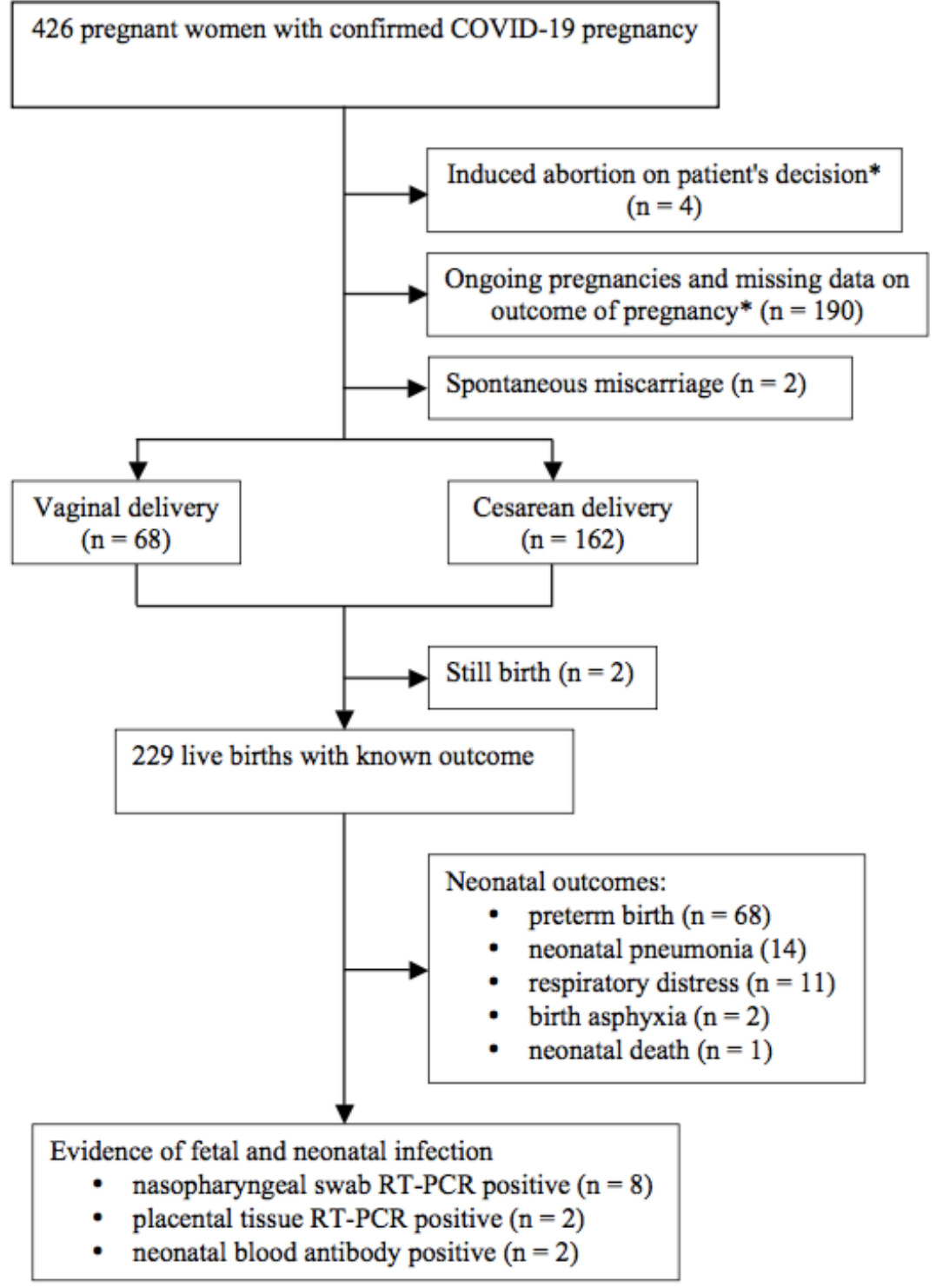

Figure 1. An outline of foetal and neonatal outcome * excluded from the study 
Table 1. Adverse outcome reported in pregnant women with COVID-19

\begin{tabular}{|lr|}
\hline Adverse outcome & Number (n) \\
\hline Miscarriage & 2 \\
Still birth & 2 \\
Preterm neonate delivery & 69 \\
NICU admission & 34 \\
Neonatal pneumonia & 14 \\
Respiratory distress syndrome & 11 \\
Birth asphyxia & 2 \\
Transient tachypnea of newborn & 1 \\
Mechanical ventilation & 7 \\
Neonatal death & 1
\end{tabular}

Maternal characteristics and mode of delivery:

Maternal age of included cases ranged from 18 to 44 years. Of 230 pregnant women with COVID-19, 48 had severe $(n=23)$ and critical $(n=25)$ disease. Fever and cough were the two most common symptoms reported by pregnant women. A threetiered disease severity category defined by $\mathrm{Wu}$ and McGooganet et al. ${ }^{25}$ was used as follows: mild ("no pneumonia and mild pneumonia"), severe ("dyspnea, respiratory rate $>30 / \mathrm{min}$, blood oxygen saturation $<93 \%$, partial pressure of arterial oxygen to fraction of inspired oxygen ratio $<300$, and/or lung infiltrates $>50 \%$ within 24 to 48 hours"), and critical ("respiratory failure, septic shock, and/or multiple organ dysfunction or failure").

All except two pregnant women were in their third trimester. Laboratory diagnosis of COVID-19 in 225 pregnant women was made before the delivery and remaining five were diagnosed within 36 hours after delivery. Amongst all deliveries, 163 (70.9\%) deliveries were full term and 67 (29.1\%) were delivered prematurely ( $<37$ weeks of gestation). Among preterm deliveries, nine were associated with premature rupture of membrane (PROM). Placental abnormality included placental abruptio in three and placenta previa in one. Intrauterine foetal distress was seen in nine pregnant women. Of all the pregnant women with COVID-19 who gave birth, $70.4 \%(n=162)$ had gone through
Table 2. Maternal and neonatal samples tested for SARS-CoV-2 and their result

\begin{tabular}{|lcrr|}
\hline Type of specimen & $\begin{array}{c}\text { Total no. } \\
\text { tested }\end{array}$ & $\begin{array}{c}\text { Positive } \\
\text { result }\end{array}$ & $\begin{array}{c}\text { \% of } \\
\text { positive } \\
\text { result }\end{array}$ \\
\hline $\begin{array}{l}\text { Neonatal naso/ } \\
\text { oropharyngeal swab }\end{array}$ & 160 & 8 & $5 \%$ \\
$\begin{array}{l}\text { Maternal vaginal } \\
\text { swab }\end{array}$ & 16 & 1 & $6.3 \%$ \\
Placenta & 13 & 2 & $15.4 \%$ \\
Breast milk & 12 & 2 & $16.7 \%$ \\
Maternal stool & 9 & 1 & $11.1 \%$ \\
Cord blood & 8 & 0 & 0 \\
Amniotic fluid & 8 & 0 & 0 \\
$\begin{array}{l}\text { Neonatal anal swab/ } \\
\text { stool }\end{array}$ & 8 & 1 & $12.5 \%$ \\
Neonatal blood & 4 & 2 & $50 \%$ \\
Fetal tissue & 2 & 0 & 0 \\
Umbilical cord & 1 & 0 & 0 \\
tissue & & & \\
\hline
\end{tabular}

cesarean section and the rest $(n=68)$ were vaginal delivery.

\section{Adverse fetal and neonatal outcomes:}

Out of 230 pregnancies with COVID-19 including three twin sets (babies, $n=233$ ), there were 229 live births, two still births and two miscarriages. Apgar score information was available for 120 live newborns with score being $>7$ in $95 \%$ of the cases. Thirteen out of 74 newborns, with birth weight record, weighed less than 2500 gm. Adverse outcome reported is summarised in table 1. Thirtyfour babies out of 229 live births were admitted and treated in NICU. ${ }^{18-21,24}$ Reasons for NICU admission were prematurity $(\mathrm{n}=21)$, RDS of prematurity $(\mathrm{n}=8), \operatorname{TTN}(\mathrm{n}=1), \operatorname{RDS}(\mathrm{n}=1)$, pneumonia $(\mathrm{n}=1)$, haemolytic anaemia $(\mathrm{n}=1)$, and recurrent hypoglycaemia $(n=1)$. Among all the neonates admitted to NICU, seven (RDS of prematurity $=6$ and pneumonia $=1$ ) were mechanically ventilated.

Baud et al. ${ }^{23}$ reported a spontaneous miscarriage in a symptomatic primigravidae at 19 week's gestation that appeared related to placental SARS-CoV-2 infection. Similarly, Wu et al. ${ }^{14}$ reported a case of missed abortion in a primigravidae with mild symptoms at $5^{+1}$ weeks of gestation. Two papers ${ }^{2,11}$ 
reported one case of still birth in each. One study ${ }^{11}$ reported a case of still birth in a 31 years old symptomatic mother at $34^{+}$week's gestation who required admission in intensive care unit (ICU) with multiple organ dysfunction syndrome (MODS) including acute respiratory distress syndrome (ARDS) prompting mechanical ventilation, acute hepatic failure, acute renal failure, and septic shock. Another study reported one still birth at $38^{+}$weeks of gestation of unknown etiology. ${ }^{2}$ Two cases of birth asphyxia were reported. One case of neonatal death following birth asphyxia was reported by $\mathrm{Li}$ et al. ${ }^{9}$ The mother of this neonate developed acute respiratory distress syndrome (ARDS) and septic shock and was admitted in intensive care unit (ICU) for mechanical ventilation. The neonate was delivered by emergency cesarean section at $35^{+}$weeks of gestation with birth asphyxia (APGAR of 1 at one minute, five minute, and 10 minute). The baby was mechanically ventilated and died at two hours of life. Respiratory distress syndrome was reported in 11 newborns by four studies..$^{15,19-21}$ Ten out of 11 newborns with respiratory distress were delivered prematurely. One of the neonates born prematurely was diagnosed as a case of TTN. ${ }^{19}$ There was one case each of hypoglycaemia ${ }^{24}$ and haemolytic anaemia. $^{20}$

Six out of 21 studies reported pneumonia in newborn babies. Amongst 14 cases $^{7,8,14,16,17,21}$ of neonatal pneumonia, three newborns tested positive for SARS-CoV-2 infection in their nasopharyngeal swabs taken one to three days after the birth. Lu et al. ${ }^{17}$ stated that three newborns were diagnosed with bacterial pneumonia based on history, laboratory investigations, and imaging studies. Fan and colleagues ${ }^{7}$ reported pneumonia in two neonates. One of them developed low grade fever, abdominal distension, and lymphopenia on third day. Chest x-ray revealed bilateral diffuse haziness. The other neonates also developed mild pneumonia and lymphopenia. Wu et al. ${ }^{14}$ reported two cases of pneumonia in premature neonates diagnosed by chest $\mathrm{x}$-ray. Yu et al. ${ }^{16}$ reported a case of neonatal pneumonia that developed mild shortness of breath after 36 hours of birth and chest x-ray revealed pulmonary infection. Ferrazzi et al. ${ }^{21}$ reported a case of severe neonatal pneumonia that had respiratory symptoms after three days and had to be mechanically ventilated for 24 hours before recovery. One study reported five cases of neonatal pneumonia without any information on clinical presentation. ${ }^{8}$ All the newborns with pneumonia recovered after treatment.

Details of type and number of samples tested and their result are described in table 2. Site of sampling of specimen for SARS-CoV-2 testing was not mentioned in two studies involving 24 neonates $^{13,18}$ and these samples were presumed to be neonatal throat swabs. In two studies, foetal tissue was tested. ${ }^{2,23}$ Of the two cases with positive breast milk testing, one of them tested negative on repeat testing after two days. In both the cases probable respiratory contamination of breast milk from the mother could not be ruled out.

In five studies, ${ }^{8,16,18,21,24}$ eight newborn babies tested positive for SARS-CoV-2 in their nasopharyngeal samples by RT-PCR. Of all positive neonates, five tested positive on first day, one at 36 hours, one at 48 hours and one after three days of delivery. Three papers ${ }^{16,21,24}$ among those newborns who tested positive reported clinical manifestations. Yu et al. ${ }^{16}$ reported one newborn that developed mild shortness of breath on second day of life. Ferrazzi et al. ${ }^{21}$ reported a neonate with gastrointestinal symptoms few hours after birth and respiratory symptoms after three days requiring mechanical ventilation for 24 hours. Kirtsman et al. ${ }^{24}$ reported a newborn with mild hypothermia, feeding difficulty and intermittent hypoglycemic episodes admitted to NICU at 37 hours for intravenous glucose, thermoregulation and supportive oral feeding. One study reported pneumonia in a neonate with no information on symptomatology whereas a case reported by Pierce-Williams et al. ${ }^{18}$ was asymptomatic. All symptomatic neonates had full recovery after treatment.

\section{DISCUSSION}

In this review we analysed foetal and neonatal outcome of 230 pregnant women with COVID-19. Data retrieved from these studies showed that infected pregnant women had higher incidence of 
cesarean delivery. More than two thirds $(70.4 \%)$ of pregnant women underwent cesarean section.

Respiratory virus infection during pregnancy seems to be associated with unfavourable outcomes like increased early trimester pregnancy loss, foetal growth restrictions, and foetal distress. ${ }^{26}$ In our review, it was not clear whether the first trimester missed abortion was related to maternal SARSCoV-2 infection or numerous other causes. However, the second trimester abortion was attributed to placental SARS-CoV-2 infection based on positive placental nucleic acid test for the virus and placental histology.

Preterm delivery was the most common adverse outcome reported followed by neonatal pneumonia. Also, prematurity was the most common cause for NICU admission. The percentage of preterm birth observed in pregnant women with COVID-19 is relatively higher $(29.6 \%)$ compared to $5 \%$ to $18 \%$ preterm birth in general population. ${ }^{27}$ Out of 48 pregnant women with severe and critical illness, 30 $(62.5 \%)$ delivered prematurely whereas rate of preterm delivery in asymptomatic and mildly symptomatic pregnant women was only $20.3 \%$ (37 out of 182). Hence the incidence of preterm delivery seems to be significantly higher in pregnant women with severe infection and critical illness.

Available studies did not find any significant difference in the rate of SARS-CoV-2 positivity among neonates born to COVID-19 confirmed mother with respect to mode of delivery. However, only small percentage of newborns were SARSCoV-2 positive in the study. In addition, one mother tested positive for the virus in vaginal secretion that would increase the chance of vertical transmission via ingestion or aspiration during vaginal delivery. So, studies with large number of neonates are needed to confirm or refute this preliminary finding.

The present studies estimate that $6.1 \%$ of all live born neonates delivered to COVID-19 mothers had pneumonia. The proportion of newborns with neonatal pneumonia in this review is comparable to that of general population with incidence of $1-10 \%$ including preterm. ${ }^{28}$ Except one, all cases of respiratory distress were associated with premature birth. Prematurity appears plausible explanation for RDS rather than the effect of maternal SARSCoV-2 infection. There was one neonatal death following birth asphyxia in critically ill mother with MODS and is attributed to rapidly deteriorating maternal condition and maternal infection associated inflammatory storm attacking on the foetal organs.

Only eight out of 161 neonates tested positive. Of these positive results, samples from five neonates were collected within first 24 hours of birth. The rest were tested between two to four days so the possibility of horizontal transmission could not be ruled out with certainty in them. Likewise, of the five neonates who tested positive on first day of life, two neonates were breast fed by their SARSCoV-2 positive mothers without using face mask. In the remaining three neonates who tested positive on first day, all were separated from their mother immediately after delivery and had not contacted their mother prior to sample collection. However, only one neonate was evaluated for other supporting evidence of vertical transmission in the case reported by Kirtsman et al. ${ }^{24}$ Samples tested with positive result for the virus included maternal vaginal swab, breast milk, placenta and neonatal serum and stool specimen. It was described as a probable case as opposed to confirmed case because of lack of detection of SARS-CoV-2 gene in the umbilical cord tissue and absence of cord blood testing for the virus. There was one more report of placental positivity in second trimester abortion reported by Baud et al. $^{23}$ that could suggest vertical transmission. However, other foetal samples including mouth swab, meconium, foetal blood and tissues were negative for SARS-CoV-2 ruling out vertical transmission. One neonate born to COVID-19 mother in the study by $\mathrm{Wu}$ and colleagues was separately reported by Dong et al. ${ }^{29}$ as a case of possible vertical transmission. The newborn had elevated IgM and IgG antibodies against SARS-CoV-2 in a blood sample drawn two hours after birth. On the contrary, the neonate's nasopharyngeal swab repeatedly tested negative for the virus on RT-PCR. PCR testing of amniotic fluid and placenta was not performed. Thus, definite evidence supporting vertical transmission of 
COVID-19 is still lacking. However, the possibility of vertical transmission cannot be refuted until it is evaluated in larger population.

Vertical transmission is a key concern for pregnant women with SARS-CoV-2 infection. Vertical transmission of infection usually occurs in utero by transfer of causative agent across the placenta or by ingestion or aspiration of maternal genital tract secretions during delivery, and in the postpartum period via breast milk. ${ }^{30}$ In these studies, samples such as placenta, amniotic fluid, cord blood, maternal vaginal secretions, breast milk, neonatal throat swab and anal swab, foetal tissue and umbilical cord tissue were tested for evidence of SARS-CoV-2 infection. These articles suggested that the possibility of vertical transmission of infection in pregnant women with COVID-19 in late pregnancy is trivial. However, these results might have been affected by small sample size, and gestational age at the time of infection.

One of the strengths of our analysis is that it includes one of largest number of cases of laboratory confirmed pregnant mothers with COVID-19. Good proportion of severe and critical cases of COVID-19 pregnant women included in this review is our next strength. One limitation of our study is that, we included half data on pregnant women with COVID-19 from China, where the obstetric and neonatal management decisions would differ. Another limitation is that availability of information regarding clinical manifestations was sparse among newborns delivered to SARSCoV-2 infected pregnant mothers.

\section{CONCLUSIONS}

Our review concludes that the majority of the adverse outcomes may be related to increased preterm delivery and cesarean section rates, both of which could have occurred in usual circumstances depending upon maternal medical or obstetric comorbidities. The current evidence suggests low rate of morbidity and much less mortality in neonates born to mothers with COVID-19. Due to the lack of complete set of evidence and difficulty in ruling out maternal contamination of foetal and neonatal samples in very limited SARS-CoV-2 positive neonates born to COVID-19 positive mothers who were evaluated for congenital infection, vertical transmission has not been proven till now. Possibility of vertical transmission still remains the area for active research as it can't be ruled out with certainty. With limited evidence regarding vertical transmission and viral shedding in genital secretions, vaginal delivery can be considered in stable patients. Furthermore, more data from larger studies across wide range of patients, population, and health care settings are needed to better assess the foetal and neonatal outcomes.

\section{REFERENCES}

1. Su S, Wong G, Shi W, Liu J, Lai ACK, Zhou J, et al. Epidemiology, Genetic Recombination, and Pathogenesis of Coronaviruses. Trends Microbiol. 2016;24(6):490-502. DOI: 10.1016/j.tim.2016.03.003.

2. Lokken EM, Walker CL, Delaney S, Kachikis A, Kretzer NM, Erickson A, et al. Clinical Characteristics of 46 Pregnant Women with a SARS-CoV-2 Infection in Washington State. Am J Obstet Gynecol. 2020;1(1):1-14. DOI: 10.1016/j.ajog.2020.05.031.

3. Goodnight WH, Soper DE. Pneumonia in pregnancy. Crit care med. 2005;33(10):390-7. DOI: 10.1097/01.ccm. 0000182483.24836 .66 .

4. Alfaraj SH, Al-Tawfiq JA, Memish ZA. Middle East Respiratory Syndrome Coronavirus (MERS-CoV) infection during pregnancy: Report of two cases and review of the literature. J Microbiol Immunol Infect. 2019;52(3):501-3. DOI: $10.1016 /$ j.jmii.2018.04.005.

5. Chen H, Guo J, Wang C, Luo F, Yu X, Zhang W, et al. Clinical characteristics and intrauterine vertical transmission potential of COVID-19 infection in nine pregnant women: a retrospective review of medical records. Lancet. 2020;395(10226):809-15. DOI: 10.1016/S0140-6736(20)30360-3.

6. Chen S, Huang B, Luo DJ, Li X, Yang F, Zhao Y, et al. Pregnancy with new coronavirus infection: clinical characteristics and placental pathological analysis of three cases. Chin J Pathol. 2020;49(5):418-23. DOI: 10.3760/ cma.j.cn112151-20200225-00138. 
7. Fan C, Lei D, Fang C, Li C, Wang M, Liu Y, et al. Perinatal Transmission of COVID-19 Associated SARS-CoV-2: Should We Worry? Clin Infect Dis. 2020;x(x):1-3. DOI: 10.1093/cid/ciaa226.

8. Khan S, Jun L, Nawsherwan A, Siddique R, Li Y, Han G, et al. Association of COVID-19 with pregnancy outcomes in health-care workers and general women. Clin Microbiol Infect. 2020;26(6):788-90. DOI: 10.1016/j.cmi. 2020.03.034.

9. Li J, Wang Y, Zeng Y, Song T, Pan X, Jia M, et al. Critically ill pregnant patient with COVID-19 and neonatal death within two hours of birth. Int J Gynaecol Obstet. 2020;150(1):1-4. DOI: 10.1002/ijgo.13189.

10. Li N, Han L, Peng M, Lv Y, Ouyang Y, Liu K, et al. Maternal and neonatal outcomes of pregnant women with COVID-19 pneumonia: a case-control study. Clin Infect Dis. 2020;xx(xx):1-8. DOI: 10.1093/cid/ciaa352.

11. Liu Y, Chen H, Tang K, Guo Y. Clinical manifestations and outcome of SARS-CoV-2 infection during pregnancy. J Infect. 2020. DOI: 10.1016/j.jinf.2020.02.028.

12. Lu D, Sang L, Du S, Li T, Chang Y, Yang XA. Asymptomatic COVID-19 infection in late pregnancy indicated no vertical transmission. J Med Virol. 2020;92(9):1-8. DOI: 10.1002/jmv.25927.

13. Qiancheng X, Jian S, Lingling P, Lei H, Xiaogan J, Weihua L, et al. Coronavirus disease 2019 in pregnancy. Int J Infect Dis. 2020;95:376-83. DOI: 10.1016/j.ijid.2020.04.065.

14. Wu Y, Liu C, Dong L, Zhang C, Chen Y, Liu J, et al. Coronavirus disease 2019 among pregnant Chinese women: Case series data on the safety of vaginal birth and breastfeeding. BJOG. 2020. DOI: 10.1111/1471-0528.16276.

15. Yang H, Sun G, Tang F, Peng M, Gao Y, Peng J, et al. Clinical features and outcomes of pregnant women suspected of coronavirus disease 2019. J Infect. 2020. DOI: 10.1016/j.jinf.2020.04.003.

16. Yu N, Li W, Kang Q, Xiong Z, Wang S, Lin X, et al. Clinical features and obstetric and neonatal outcomes of pregnant patients with COVID-19 in Wuhan, China: a retrospective, single-centre, descriptive study. Lancet Infect Dis. 2020;20(5):559-64. DOI: 10.1016/S1473-3099(20)30176-6.

17. Zhang L, Jiang Y, Wei M, Cheng BH, Zhou XC, Li J, et al. Analysis of the pregnancy outcomes in pregnant women with COVID-19 in Hubei Province. Zhonghua Fu Chan Ke Za Zhi. 2020;55(3):166-71. DOI: 10.3760/ cma.j.cn112141-20200218-00111.

18. Pierce-Williams RAM, Burd J, Felder L, Khoury R, Bernstein PS, Avila K, et al. Clinical course of severe and critical COVID-19 in hospitalized pregnancies: a US cohort study. Am J Obstet Gynecol MFM. 2020:100134. DOI: 10.1016/j.ajogmf.2020.100134

19. Romagano MP, Guerrero K, Spillane N, Kayaalp E, Smilen SW, Alvarez M, et al. Perinatal outcome in critically ill pregnant women with COVID-19. Am J Obstet Gynecol. 2020. DOI: https://doi.org/10.1016/j.ajogmf.2020.100151.

20. Pereira A, Cruz-Melguizo S, Adrien M, Fuentes L, Marin E, Perez-Medina T. Clinical course of Coronavirus Disease-2019 (COVID-19) in pregnancy. Acta Obstet Gynecol Scand. 2020. DOI: 10.1111/aogs.13921.

21. Ferrazzi E, Frigerio L, Savasi V, Vergani P, Prefumo F, Barresi S, et al. Vaginal delivery in SARS-CoV-2 infected pregnant women in Northern Italy: a retrospective analysis. BJOG. 2020. DOI: 10.1111/1471-0528.16278.

22. Lyra J, Valente R, Rosario M, Guimaraes M. Cesarean Section in a Pregnant Woman with COVID-19: First Case in Portugal. Acta Med Port. 2020. DOI: 10.20344/amp.13883.

23. Baud D, Greub G, Favre G, Gengler C, Jaton K, Dubruc E, et al. Second-Trimester Miscarriage in a Pregnant Woman With SARS-CoV-2 Infection. JAMA. 2020. DOI: 10.1001/jama.2020.7233.

24. Kirtsman M, Diambomba Y, Poutanen SM, Malinowski AK, Vlachodimitropoulou E, Parks WT, et al. Probable congenital SARS-CoV-2 infection in a neonate born to a woman with active SARS-CoV-2 infection. CMAJ. 2020. DOI: $10.1503 / \mathrm{cmaj} .200821$.

25. Wu Z, McGoogan JM. Characteristics of and Important Lessons From the Coronavirus Disease 2019 (COVID-19) Outbreak in China: Summary of a Report of 72314 Cases From the Chinese Centre for Disease Control and Prevention. JAMA. 2020. DOI: 10.1001/jama.2020.2648.

26. Rasmussen SA, Jamieson DJ. Coronavirus Disease 2019 (COVID-19) and Pregnancy: Responding to a Rapidly Evolving Situation. Obstet Gynecol. 2020;135(5):999-1002. DOI: 10.1097/AOG.0000000000003873.

27. Fact sheet: Preterm birth [Internet]: World Health Organisation; 2018 Feb.19 [cited 2020 Jun 30]. Available from: https://www.who.int/news-room/fact-sheets/detail/preterm-birth. 
28. Vankaam AHLC. Neonatal Mechanical Ventilation. Mechanical Ventilation: Clinical Applications and Pathophysiology. 1st ed. Philadelphia: W.B. Saunders; 2008. p. 529-51.

29. Dong L, Tian J, He S, Zhu C, Wang J, Liu C, et al. Possible Vertical Transmission of SARS-CoV-2 From an Infected Mother to Her Newborn. JAMA. 2020. DOI: 10.1001/jama.2020.4621.

30. Rasmussen SA, Smulian JC, Lednicky JA, Wen TS, Jamieson DJ. Coronavirus Disease 2019 (COVID-19) and pregnancy: what obstetricians need to know. Am J Obstet Gynecol. 2020;222(5):415-26. DOI: 10.1016/j.ajog. 2020.02.017. 